\title{
Classification of string links up to self delta-moves and concordance
}

\author{
AKIRA YASUHARA
}

\begin{abstract}
For an $n$-component string link, the Milnor's concordance invariant is defined for each sequence $I=i_{1} i_{2} \cdots i_{m}\left(i_{j} \in\{1, \ldots, n\}\right)$. Let $r(I)$ denote the maximum number of times that any index appears. We show that two string links are equivalent up to self $\Delta$-moves and concordance if and only if their Milnor invariants coincide for all sequences $I$ with $r(I) \leq 2$.
\end{abstract}

$57 \mathrm{M} 25,57 \mathrm{M} 27$

\section{Introduction}

For an $n$-component link $L$, the Milnor $\bar{\mu}$-invariant $\bar{\mu}_{L}(I)$ is defined for each sequence $I=i_{1} i_{2} \cdots i_{m}\left(i_{j} \in\{1, \ldots, n\}\right)$; see Milnor [13; 14]. Let $r(I)$ denote the maximum number of times that any index appears. For example, $r(1123)=2$ and $r(1231223)=3$. It is known that if $r(I)=1$, then $\bar{\mu}_{L}(I)$ is a link-homotopy invariant [13], where link-homotopy is an equivalence relation on links generated by self crossing changes. Similarly, for a string link $L$, the Milnor $\mu$-invariant $\mu_{L}(I)$ is defined; see Habegger and Lin [7]. Milnor $\mu$-invariants give a link-homotopy classification for string links.

Theorem 1.1 [7] Two $n$-component string links $L$ and $L^{\prime}$ are link-homotopic if and only if $\mu_{L}(I)=\mu_{L^{\prime}}(I)$ for any $I$ with $r(I)=1$.

Theorem 1.1 implies the following.

Theorem 1.2 [13; 7] A link $L$ in $S^{3}$ is link-homotopic to the trivial link if and only if $\bar{\mu}_{L}(I)=0$ for any $I$ with $r(I)=1$.

Although Milnor invariants for sequences $I$ with $r(I) \geq 2$ are not necessarily linkhomotopy invariants, they are generalized link-homotopy invariants. In fact, T Fleming and the author [4, Theorem 1.1] showed that $\bar{\mu}$-invariants for sequences $I$ with $r(I) \leq k$ are self $C_{k}$-equivalence invariants of links in $S^{3}$, where the self $C_{k}$-equivalence is an equivalence relation on (string) links generated by self $C_{k}$-moves, and a $C_{k}$-move is 
a local move on links defined by Habiro $[9 ; 10]$. This statement holds for $\mu$-invariants of string links as well. The proof is the same as the one of [4, Theorem 1.1] except for using Proposition 3.1 instead of [14, Theorem 7]. The link-homotopy coincides with the self $C_{1}$-equivalence. The self $C_{2}$-equivalence coincides with the self $\Delta$-equivalence, which is an equivalence relation generated by self $\Delta$-moves. A $\Delta$-move is a local move as illustrated in Figure 1; see Murakami and Nakanishi [15]. The $\Delta-$ move is called a self $\Delta$-move if all strands in Figure 1 belong to the same component of a (string) link; see Shibuya [19].

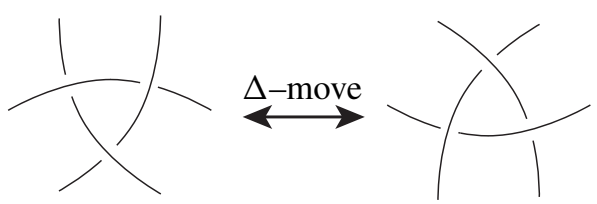

Figure 1

A self $\Delta$-equivalence classification of 2-component links was given by Y Nakanishi and Y Ohyama [16] and for 2-component string links by Fleming and the author [3]. It is still open for (string) links with at least 3 components.

The following result is a generalization of Theorem 1.2.

Theorem 1.3 [23, Corollary 1.5] A link $L$ is self $\Delta$-equivalent to the trivial link if and only if $\bar{\mu}_{L}(I)=0$ for any $I$ with $r(I) \leq 2$.

In this paper, we generalize Theorem 1.1 and give a certain geometric characterization for string links whose $\mu$-invariants coincide for all sequences $I$ with $r(I) \leq 2$. It is known that self $\Delta$-equivalence is too fine to give the characterization, ie, there are 2-component string links such that their $\mu$-invariants vanish for all sequences $I$ with $r(I) \leq 2$ and they are not self $\Delta$-equivalent to the trivial string link [3].

Since Milnor invariants are concordance invariants by Casson [1] and concordance does not imply self $\Delta$-equivalence by Nakanishi and Shibuya [17] and Nakanishi, Shibuya and Yasuhara [18], an equivalence relation generated by concordance and self $\Delta$-moves is looser than the self $\Delta$-equivalence and preserves Milnor invariants for all sequences $I$ with $r(I) \leq 2$. We define the equivalence relation as follows. Two (string) links $L$ and $L^{\prime}$ are self- $\Delta$ concordant if there is a sequence $L=L_{1}, \ldots, L_{m}=L^{\prime}$ of (string) links such that for each $i(\in\{1, \ldots, m-1\}), L_{i}$ and $L_{i+1}$ are either concordant or self $\Delta$-equivalent. 
The following is the main result of this paper.

Theorem 1.4 Two $n$-component string links $L$ and $L^{\prime}$ are self- $\Delta$ concordant if and only if $\mu_{L}(I)=\mu_{L^{\prime}}(I)$ for any $I$ with $r(I) \leq 2$.

For an $n$-component string link $L$, let $L(k)$ be a $k n$-component string link obtained from $L$ by replacing each component of $L$ with $k$ zero framed parallels of it. By combining Theorem 1.4 and Proposition 3.2, we have the following corollary.

Corollary 1.5 Two string links $L$ and $L^{\prime}$ are self- $\Delta$ concordant if and only if $L(2)$ and $L^{\prime}(2)$ are link-homotopic.

Let $\mathcal{S} \mathcal{L}(n)$ be the set of $n$-component string links, and let $\mathcal{S} \mathcal{L}(n) /(\mathrm{s} \Delta+\mathrm{c})$ (resp. $\mathcal{S L}(n) / C_{m}$ ) be the set of self- $\Delta$ concordance classes (resp. the set of $C_{m}$-equivalence classes). Habiro showed that $\mathcal{S} \mathcal{L}(n) / C_{m}$ is a nilpotent group [10, Theorem 5.4]. Since $C_{2 n}$-equivalence for $n$-component (string) links implies self $\Delta$-equivalence [4, Lemma 1.2], we have that $\mathcal{S} \mathcal{L}(n) /(\mathrm{s} \Delta+\mathrm{c})$ is a nilpotent group. Moreover, since the first nonvanishing $\mu$-invariants are additive under the stacking product (for example see Cochran [2] and Habegger and Masbaum [8]), by Theorem 1.4, we have the following proposition.

Proposition 1.6 The quotient $\mathcal{S} \mathcal{L}(n) /(\mathrm{s} \Delta+\mathrm{c})$ forms a torsion-free nilpotent group under the stacking product.

Acknowledgments The author would like to thank Professor Nathan Habegger and Professor Uwe Kaiser for many useful discussions which inspired him to consider the equivalence relation generated by self $\Delta$-moves and concordance. He is also very grateful to Professor Jonathan Hillman for helpful comments. He is partially supported by a Grant-in-Aid for Scientific Research (C) (\#20540065) of the Japan Society for the Promotion of Science.

\section{String links and Milnor invariants}

In this section, we summarize the definitions of string links and Milnor invariants of links and string links.

A string link is a generalization of a pure braid defined by Habegger and Lin [7]. 


\subsection{String links}

Let $D$ be the unit disk in the plane and let $I=[0,1]$ be the unit interval. Choose $n$ points $p_{1}, \ldots, p_{n}$ in the interior of $D$ so that $p_{1}, \ldots, p_{n}$ lie in order on the $x$-axis, see Figure 2. An $n$-component string link $L=K_{1} \cup \cdots \cup K_{n}$ in $D \times I$ is a disjoint union of oriented $\operatorname{arcs} K_{1}, \ldots, K_{n}$ such that each $K_{i}$ runs from $\left(p_{i}, 0\right)$ to $\left(p_{i}, 1\right)$ $(i=1, \ldots, n)$. A string link $K_{1} \cup \cdots \cup K_{n}$ with $K_{i}=\left\{p_{i}\right\} \times I(i=1, \ldots, n)$ is called the $n$-component trivial string link and denoted by $\mathbf{1}_{n}$. For a string link $L$ in $D \times I$, the closure $\operatorname{cl}(L)$ of $L$ is a link in $S^{3}$ obtained from $L$ by identifying points of $\partial(D \times I)$ with their images under the projection $D \times I \longrightarrow D$. It is easy to see that every link is the closure of some string link.

Milnor defined in [13; 14] a family of invariants of oriented, ordered links in $S^{3}$, known as Milnor $\bar{\mu}$-invariants.

\subsection{Milnor invariants of links}

Given an $n$-component link $L=K_{1} \cup \cdots \cup K_{n}$ in $S^{3}$, denote by $G$ the fundamental group of $S^{3} \backslash L$, and by $G_{q}$ the $q$-th subgroup of the lower central series of $G$. We have a presentation of $G / G_{q}$ with $n$ generators, given by a meridian $\alpha_{i}$ of each component $K_{i}$. So, for each $j \in\{1, \ldots, n\}$, the longitude $l_{j}$ of the $j$-th component of $L$ is expressed modulo $G_{q}$ as a word in the $\alpha_{i}$ 's (abusing notation, we still denote this word by $l_{j}$ ). The Magnus expansion $E\left(l_{j}\right)$ of $l_{j}$ is the formal power series in noncommuting variables $X_{1}, \ldots, X_{n}$ obtained by substituting $1+X_{i}$ for $\alpha_{i}$ and $1-X_{i}+X_{i}^{2}-X_{i}^{3}+\cdots$ for $\alpha_{i}^{-1}, i=1, \ldots, n$. Let $I=i_{1} i_{2} \cdots i_{k-1} j(k \leq q)$ be a sequence in $\{1, \ldots, n\}$. Denote by $\mu_{L}(I)$ the coefficient of $X_{i_{1}} \cdots X_{i_{k-1}}$ in the Magnus expansion $E\left(l_{j}\right)$. Milnor $\bar{\mu}$-invariant $\bar{\mu} L(I)$ is the residue class of $\mu_{L}(I)$ modulo the greatest common divisor of all $\mu_{L}(J)$ such that $J$ is obtained from $I$ by removing at least one index, and permutating the remaining indices cyclicly.

In [7], Habegger and Lin define the Milnor invariants of string links. We also refer the reader to Habegger and Masbaum [8].

\subsection{Milnor invariants of string links}

In the unit disk $D$, we chose a point $e \in \partial D$ and loops $\alpha_{1}, \ldots, \alpha_{n}$ as illustrated in Figure 2. For an $n$-component string link $L=K_{1} \cup \cdots \cup K_{n}$ in $D \times I$ with $\partial K_{j}=$ $\left\{\left(p_{j}, 0\right),\left(p_{j}, 1\right)\right\}(j=1, \ldots, n)$, set $Y=(D \times I) \backslash L, Y_{0}=(D \times\{0\}) \backslash L$, and $Y_{1}=(D \times$ $\{1\}) \backslash L$. We may assume that each $\pi_{1}\left(Y_{t}\right)(t \in\{0,1\})$ with base point $(e, t)$ is the free group $F(n)$ on generators $\alpha_{1}, \ldots, \alpha_{n}$. We denote the image of $\alpha_{j}$ in the lower central series quotient $F(n) / F(n)_{q}$ again by $\alpha_{j}$. By Stallings' theorem [22], the inclusions 


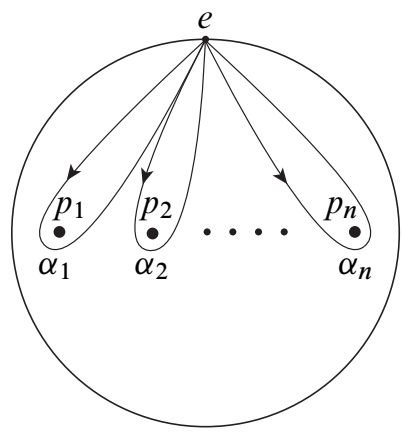

Figure 2

$i_{t}: Y_{t} \longrightarrow Y$ induce isomorphisms $\left(i_{t}\right)_{*}: \pi_{1}\left(Y_{t}\right) / \pi_{1}\left(Y_{t}\right)_{q} \longrightarrow \pi_{1}(Y) / \pi_{1}(Y)_{q}$ for any positive integer $q$. Hence the induced map $\left(i_{1}\right)_{*}^{-1} \circ\left(i_{0}\right)_{*}$ is an automorphism of $F(n) / F(n)_{q}$ and sends each $\alpha_{j}$ to a conjugate $l_{j} \alpha_{j} l_{j}^{-1}$ of $\alpha_{j}$, where $l_{j}$ is the longitude of $K_{j}$ defined as follows. Let $\gamma_{j}$ be a zero framed parallel of $K_{j}$ such that the endpoints $\left(c_{j}, t\right) \in D \times\{t\}(t=0,1)$ lie on the $x$-axis in $\mathbb{R}^{2} \times\{t\}$. The longitude $l_{j} \in F(n) / F(n)_{q}$ is an element represented by the union of the arc $\gamma_{j}$ and the segments $e \times I, c_{j} e \times\{0,1\}$ under $\left(i_{1}\right)_{*}^{-1}$. The coefficient $\mu_{L}\left(i_{1} i_{2} \cdots i_{k-1} j\right)(k \leq q)$ of $X_{i_{1}} \cdots X_{i_{k-1}}$ in the Magnus expansion $E\left(l_{j}\right)$ is well-defined invariant of $L$, and it is called a Milnor $\mu$-invariant of $L$.

\section{Proof of Theorem 1.4}

By an argument similar to that in the proof of [14, Theorem 7], we have the following proposition.

Proposition 3.1 (cf [14, Theorem 7]) Let $L_{j}^{\prime}(j=1,2)$ be an $l$-component string link obtained from an $n$-component string link $L_{j}$ by replacing each component of $L_{j}$ with zero framed parallels of it. Suppose that the $i$-th components of $L_{1}^{\prime}$ and $L_{2}^{\prime}$ correspond to the $h(i)$-th components of $L_{1}$ and $L_{2}$ respectively. For a sequence $i_{1} i_{2} \cdots i_{m}$ of integers in $\{1,2, \ldots, l\}, \mu_{L_{1}^{\prime}}(I)=\mu_{L_{2}^{\prime}}(I)$ for any subsequence $I$ of $i_{1} i_{2} \cdots i_{m}$ if and only if $\mu_{L_{1}}(J)=\mu_{L_{2}}(J)$ for any subsequence $J$ of $h\left(i_{1}\right) h\left(i_{2}\right) \cdots h\left(i_{m}\right)$.

Remark It is shown that for a link $L^{\prime}$ in $S^{3}$ obtained from a link $L$ by taking zero framed parallels of the components of $L$, if the $i$-th component of $L^{\prime}$ corresponds to the $h(i)$-th component of $L$, then $\bar{\mu}_{L^{\prime}}\left(i_{1} i_{2} \cdots i_{m}\right)=\bar{\mu}_{L}\left(h\left(i_{1}\right) h\left(i_{2}\right) \cdots h\left(i_{m}\right)\right)[14$, Theorem 7]. Although this looks stronger than the proposition above, it holds for the residue class $\bar{\mu}$. It does not hold for $\mu$-invariant of string links. In fact, there is the 
following example: Let $L$ be the 2-component string link illustrated in Figure 3 and $L^{\prime}$ the 3-component string link illustrated in Figure 3, which is obtained from $L$ by taking two zero framed parallels of the first component. Note that $h(1)=h(2)=1$

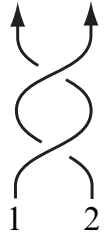

$L$

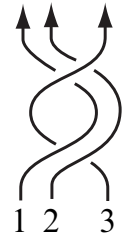

$L^{\prime}$

Figure 3

and $h(3)=2$. Then the Magnus expansion of the 2 nd longitude of $L$ is $1+X_{1}$ and the expansion of the 3 rd longitude of $L^{\prime}$ is $1+X_{2}+X_{1}+X_{1} X_{2}$. Hence we have $\mu_{L}(112) \neq \mu_{L^{\prime}}(123)$.

Proof It is enough to consider the special case where $L_{j}^{\prime}(j=1,2)$ is an $(n+1)-$ component string link obtained from an $n$-component string link $L_{j}$ by replacing the $n$-th component of $L_{j}$ with two parallels of it. We may assume that the two parallels are contained in a tubular neighborhood $N_{j}$ of the $n$-th component of $L_{j}$. Then there is the natural homomorphism from $\pi_{1}\left(E_{j}\right)\left(\cong \pi_{1}\left(E_{j} \backslash N_{j}\right)\right)$ to $\pi_{1}\left(E_{j}^{\prime}\right)$, where $E_{j}$ and $E_{j}^{\prime}$ are the complements of $L_{j}$ and $L_{j}^{\prime}$ respectively. The $i$-th longitudes $l_{j i}(i=1, \ldots, n)$ of $L_{j}$ map to the $i$-th longitudes $l_{j i}^{\prime}$ of $L_{j}^{\prime}$, the $i$-th meridians $\alpha_{j i}(i=1, \ldots, n-1)$ of $L_{j}$ map to the $i$-th meridians $\alpha_{j i}^{\prime}$ of $L_{j}^{\prime}$, and the $n$-th meridian $\alpha_{j n}$ maps to $\alpha_{j n}^{\prime} \alpha_{j n+1}^{\prime}$. Note that $l_{j n+1}^{\prime}$ is equal to $l_{j n}^{\prime}$. The Magnus expansion $M\left(l_{j i}^{\prime}\right)$ of $l_{j i}^{\prime}$ can be obtained from the expansion

$$
M\left(l_{j h(i)}\right)=1+\sum \mu_{L_{j}}\left(h_{1} \cdots h_{s} h(i)\right) X_{h_{1}} \cdots X_{h_{s}}
$$

by substituting $M\left(\alpha_{j n}^{\prime} \alpha_{j n+1}^{\prime}\right)-1=X_{n}+X_{n+1}+X_{n} X_{n+1}$ for $X_{n}$.

Hence, if $\mu_{L_{1}}(J)=\mu_{L_{2}}(J)$ for any subsequence $J$ of $h\left(i_{1}\right) \cdots h\left(i_{m}\right)$, then $\mu_{L_{1}^{\prime}}(I)=$ $\mu_{L_{2}^{\prime}}(I)$ for any subsequence of $i_{1} \cdots i_{m}$. Recall that $h(i)=i(i=1, \ldots, n-1)$ and $h(n)=h(n+1)=n$.

On the other hand, suppose that there is a subsequence $J h$ of $h\left(i_{1}\right) \cdots h\left(i_{m}\right)$ such that $\mu_{L_{1}}(J h) \neq \mu_{L_{2}}(J h)$. We may assume that the length of $J$ is minimal among all such subsequences, ie, for any subsequence $J^{\prime}(\neq J)$ of $J, \mu_{L_{1}}\left(J^{\prime} h\right)=\mu_{L_{2}}\left(J^{\prime} h\right)$. Let $k_{1} \cdots k_{t} k$ be a subsequence of $i_{1} \cdots i_{m}$ with $h\left(k_{1}\right) \cdots h\left(k_{t}\right)=J$ and $h(k)=h$. Note that $\mu_{L_{j}^{\prime}}\left(k_{1} \cdots k_{t} k\right)$ might not be equal to $\mu_{L_{j}}(J h)$ (if $k_{1} \cdots k_{t}$ contains the 
pattern $n(n+1))$. Since the Magnus expansion $M\left(l_{j k}^{\prime}\right)$ can be obtained from $M\left(l_{j h}\right)$ by substituting $X_{n}+X_{n+1}+X_{n} X_{n+1}$ for $X_{n}$, there is a set $S_{J}$, possibly $S_{J}=\{J\}$, of subsequences of $J$ such that

$$
\mu_{L_{j}^{\prime}}\left(k_{1} \cdots k_{t} k\right)=\sum_{J^{\prime} \in S_{J}} \mu_{L_{j}}\left(J^{\prime} h\right)(j=1,2) .
$$

The minimality of $J$ implies

$$
\mu_{L_{1}^{\prime}}\left(k_{1} \cdots k_{t} k\right)-\mu_{L_{2}^{\prime}}\left(k_{1} \cdots k_{t} k\right)=\mu_{L_{1}}(J h)-\mu_{L_{2}}(J h) \neq 0 .
$$

This completes the proof.

By combining Theorem 1.1 and Proposition 3.1, we have the following characterization for string links whose $\mu$-invariants coincide for all sequences $I$ with $r(I) \leq k$.

Proposition 3.2 (cf [2, Proposition 9.3]) Let $L_{1}$ and $L_{2}$ be $n$-component string links and $k$ a natural number. Let $L_{j}(k)$ be a $k n$-component string link obtained from $L_{j}$ by replacing each component of $L_{j}$ with $k$ zero framed parallels of it $(j=1,2)$. Then the following are equivalent:

(1) $\mu_{L_{1}}(J)=\mu_{L_{2}}(J)$ for any $J$ with $r(J) \leq k$.

(2) $\mu_{L_{1}(k)}(I)=\mu_{L_{2}(k)}(I)$ for any $I$ with $r(I)=1$.

(3) $L_{1}(k)$ and $L_{2}(k)$ are link-homotopic.

Proof Theorem 1.1 implies “(2) $\Leftrightarrow$ (3)". We only need to show “(1) $\Leftrightarrow(2)$ ”.

Suppose that the $i$-th components of $L_{1}(k)$ and $L_{2}(k)$ correspond to the $h(i)$-th components of $L_{1}$ and $L_{2}$ respectively. For a sequence $I=i_{1} i_{2} \cdots i_{m}$ of integers in $\{1,2, \ldots, n k\}$, let $h(I)$ denote $h\left(i_{1}\right) h\left(i_{2}\right) \cdots h\left(i_{m}\right)$.

$(1) \Rightarrow(2) \quad$ Let $I$ be a sequence of integers in $\{1,2, \ldots, n k\}$ with $r(I)=1$. Since $r(h(I)) \leq k$, for any subsequence $J$ of $h(I)$, we have $r(J) \leq k$, and hence $\mu_{L_{1}}(J)=$ $\mu_{L_{2}}(J)$. By Proposition 3.1, we have $\mu_{L_{1}(k)}(I)=\mu_{L_{2}(k)}(I)$.

(2) $\Rightarrow$ (1) Let $J$ be a sequence of integers in $\{1,2, \ldots, n\}$ with $r(J) \leq k$. Then there is a sequence $I^{\prime}$ of integers in $\{1,2, \ldots, n k\}$ with $r\left(I^{\prime}\right)=1$ and $h\left(I^{\prime}\right)=J$. Since any subsequence $I$ of $I^{\prime}$ satisfies $r(I)=1, \mu_{L_{1}(k)}(I)=\mu_{L_{2}(k)}(I)$. By Proposition 3.1 , we have $\mu_{L_{1}}(J)=\mu_{L_{2}}(J)$. 
By using Proposition 3.2, we have the following proposition.

Proposition 3.3 Let $L$ and $L^{\prime}$ be $n$-component string links and $k$ a natural number. Then $\mu_{L}(I)=\mu_{L^{\prime}}(I)$ for any $I$ with $r(I) \leq k$ if and only if $\mu_{L * \overline{L^{\prime}}}(I)=0$ for any $I$ with $r(I) \leq k$, where $*$ is the stacking product and $\overline{L^{\prime}}$ is the horizontal mirror image of $L^{\prime}$ with the orientation reversed.

Note that, for a string link $L, \bar{L}$ is the inverse of $L$ under the concordance, ie, both $\bar{L} * L$ and $L * \bar{L}$ are concordant to a trivial string link.

Proof By Proposition 3.2, $\mu_{L}(I)=\mu_{L^{\prime}}(I)$ (resp. $\left.\mu_{L * \overline{L^{\prime}}}(I)=0\right)$ for any $I$ with $r(I) \leq k$ if and only if $L(k)$ and $L^{\prime}(k)$ (resp. $\left(L * \overline{L^{\prime}}\right)(k)$ and the $k n$-component trivial string link $\mathbf{1}_{k n}$ ) are link-homotopic. Hence it is enough to show that $L(k)$ and $L^{\prime}(k)$ are link-homotopic if and only if $\left(L * \overline{L^{\prime}}\right)(k)$ and $\mathbf{1}_{k n}$ are link-homotopic. Note that $\left(L * \overline{L^{\prime}}\right)(k)=L(k) * \overline{L^{\prime}(k)}$.

If $L(k)$ and $L^{\prime}(k)$ are link-homotopic, then $L(k) * \overline{L^{\prime}(k)}$ is link-homotopic to $L^{\prime}(k) *$ $\overline{L^{\prime}(k)}$, which is concordant to $\mathbf{1}_{k n}$. Since concordance of string links implies linkhomotopy $[5 ; 6]^{1}, L(k) * \overline{L^{\prime}(k)}$ is link-homotopic to $\mathbf{1}_{k n}$.

If $L(k) * \overline{L^{\prime}(k)}$ is link-homotopic to $\mathbf{1}_{k n}$, then $L(k) * \overline{L^{\prime}(k)} * L^{\prime}(k)$ is link-homotopic to $L^{\prime}(k)$. Since $L(k) * \overline{L^{\prime}(k)} * L^{\prime}(k)$ is concordant to $L(k), L(k)$ is link-homotopic to $L^{\prime}(k)$. This completes the proof.

Two $n$-component string links $L$ and $L^{\prime}$ are weak self $\Delta$-equivalent if the closure $\operatorname{cl}\left(L * \overline{L^{\prime}}\right)$ is self $\Delta$-equivalent to the trivial link.

T Shibuya defined weak self $\Delta$-equivalence for links in $S^{3}$ [21], and showed that two links in $S^{3}$ are weak self $\Delta$-equivalent if and only if they are self- $\Delta$ concordant [20]. (In [21] and [20], the self- $\Delta$ concordance is called the $\Delta$-cobordism.) Here we give the same result for string links.

Proposition 3.4 (cf [20, Theorem]) Two string links $L$ and $L^{\prime}$ are weak self $\Delta-$ equivalent if and only if they are self- $\Delta$ concordant.

Before proving the proposition above, we need some preparation.

Let $L=K_{1} \cup \cdots \cup K_{n}$ be an $n$-component (string) link and $b$ a band attaching a single component $K_{i}$ with coherent orientation, ie, $b \cap L=b \cap K_{i} \subset \partial b$ consists

${ }^{1}$ In [5; 6], it was shown that concordance of links in $S^{3}$ implies link-homotopy. It still holds for string links. It also follows from Theorem 1.1 since Milnor invariants are concordance invariants. 
of two arcs whose orientations from $K_{i}$ are opposite to those from $\partial b$. Then $L^{\prime}=$ $(L \cup \partial b) \backslash \operatorname{int}\left(b \cap K_{i}\right)$, which is a union of an $n$-component (string) link and a knot, is said to be obtained from $L$ by fission (along a band $b$ ), and conversely $L$ is said to be obtained from $L^{\prime}$ by fusion [12].

Lemma 3.5 Let $L_{1}, L_{2}, L_{3}$ be oriented tangles such that $L_{2}$ is obtained from $L_{1}$ by a single (self) $\Delta$-move, and that $L_{3}$ is obtained from $L_{2}$ by a single fusion. Then there is an oriented tangle $L_{2}^{\prime}$ such that $L_{2}^{\prime}$ is obtained from $L_{1}$ by a single fusion, and that $L_{3}$ is obtained from $L_{2}^{\prime}$ by a single (self) $\Delta$-move.

Proof Let $B$ be a 3-ball such that $L_{1} \backslash B=L_{2} \backslash B$, and that the pair of tangles $\left(B, L_{1} \cap B\right)$ and $\left(B, L_{2} \cap B\right)$ is a (self) $\Delta$-move. Let $b$ be a fusion band with $L_{3}=\left(L_{2} \cup \partial b\right) \backslash \operatorname{int}\left(b \cap L_{2}\right)$. If $b$ intersects $B$, then we can move it out of $B$ by an isotopy fixing $L_{2}$ since $\left(B, L_{2} \cap B\right)$ is a trivial tangle. Thus we may assume that $b$ is contained in $L_{1} \backslash B$. Let $L_{2}^{\prime}$ be a link obtained from $L_{1}$ by fusion along $b$. Then $L_{3}$ is obtained from $L_{2}^{\prime}$ by a (self) $\Delta$-move, which corresponds to substituting $\left(B, L_{2} \cap B\right)$ for $\left(B, L_{1} \cap B\right)$.

Proof of Proposition 3.4 If $L$ and $L^{\prime}$ are self- $\Delta$ concordant, then $\mu_{L}(I)=\mu_{L^{\prime}}(I)$ for any $I$ with $r(I) \leq 2[1 ; 4]$. Proposition 3.3 and Theorem 1.3 imply that $\operatorname{cl}\left(L * \overline{L^{\prime}}\right)$ is self $\Delta$-equivalent to the trivial link.

Suppose $L$ and $L^{\prime}$ are weak self $\Delta$-equivalent. Since $L$ is concordant to $L * \overline{L^{\prime}} * L^{\prime}$, it is enough to show that $L * \overline{L^{\prime}} * L^{\prime}$ and $L^{\prime}$ are self $-\Delta$ concordant. The split sum of $L^{\prime}$ and $\operatorname{cl}\left(L * \overline{L^{\prime}}\right)$ is obtained from $L * \overline{L^{\prime}} * L^{\prime}$ by a finite sequence of fission, and $\operatorname{cl}\left(L * \overline{L^{\prime}}\right)$ is self $\Delta$-equivalent to the trivial link $O$. So $L * \overline{L^{\prime}} * L^{\prime}$ is obtained from the split sum of $L^{\prime}$ and $O$ by a sequence of self $\Delta$-moves and fusion. By Lemma 3.5, we can freely choose to perform all fusion first, and then all self $\Delta$-moves. Hence we have that the fusion of $L^{\prime}$ and $O$, which is concordant to $L^{\prime}$, is self $\Delta$-equivalent to $L * \overline{L^{\prime}} * L^{\prime}$.

Now we are ready to prove Theorem 1.4.

Proof of Theorem 1.4 By Theorem 1.3, $L$ and $L^{\prime}$ are weak self $\Delta$-equivalent if and only if $\bar{\mu}_{\mathrm{cl}\left(L_{1} * \overline{L^{\prime}}\right)}(I)=\mu_{L_{1} * \overline{L^{\prime}}}(I)=0$ for any $I$ with $r(I) \leq 2$. Proposition 3.3 and Proposition 3.4 complete the proof.

Remark Although the $C_{k}$-move ( $k \geq 3$ ) is not an unknotting operation, it might be reasonable to consider the following question: For two string links $L$ and $L^{\prime}$ whose components are trivial, if $\mu_{L}(I)=\mu_{L^{\prime}}(I)$ for any $I$ with $r(I) \leq k$, then are $L$ and $L^{\prime}$ 
equivalent up to self $C_{k}$-move and concordance? The question is still open, but the answer is likely negative. For example, the Hopf link with both components Whitehead doubled, which is a boundary link and thus all its Milnor invariants vanish, is neither self $C_{3}$-equivalent [4] nor concordant [11, Section 7.3] to the trivial link.

\section{References}

[1] A J Casson, Link cobordism and Milnor's invariant, Bull. London Math. Soc. 7 (1975) 39-40 MR0362286

[2] T D Cochran, Derivatives of links: Milnor's concordance invariants and Massey's products, Mem. Amer. Math. Soc. 84 (1990) x+73 MR1042041

[3] T Fleming, A Yasuhara, Milnor numbers and the self delta classification of 2-string links, from: "Intelligence of low dimensional topology 2006", (J S Carter, S Kamada, L H Kauffman, A Kawauchi, T Kohno, editors), Ser. Knots Everything 40, World Sci. Publ., Hackensack, NJ (2007) 27-34 MR2371705

[4] T Fleming, A Yasuhara, Milnor's invariants and self $C_{k}$-equivalence, Proc. Amer. Math. Soc. 137 (2009) 761-770 MR2448599

[5] C H Giffen, Link concordance implies link homotopy, Math. Scand. 45 (1979) 243-254 MR580602

[6] D L Goldsmith, Concordance implies homotopy for classical links in $M^{3}$, Comment. Math. Helv. 54 (1979) 347-355 MR543335

[7] N Habegger, X-S Lin, The classification of links up to link-homotopy, J. Amer. Math. Soc. 3 (1990) 389-419 MR1026062

[8] N Habegger, G Masbaum, The Kontsevich integral and Milnor's invariants, Topology 39 (2000) 1253-1289 MR1783857

[9] K Habiro, Aru musubime no kyokusyo sousa no zoku ni tuite, Master's thesis, University of Tokyo (1994) In Japanese

[10] K Habiro, Claspers and finite type invariants of links, Geom. Topol. 4 (2000) 1-83 MR1735632

[11] J Hillman, Algebraic invariants of links, Series on Knots and Everything 32, World Sci. Publ., River Edge, NJ (2002) MR1932169

[12] A Kawauchi, T Shibuya, S Suzuki, Descriptions on surfaces in four-space. I. Normal forms, Math. Sem. Notes Kobe Univ. 10 (1982) 75-125 MR672939

[13] J Milnor, Link groups, Ann. of Math. (2) 59 (1954) 177-195 MR0071020

[14] J Milnor, Isotopy of links. Algebraic geometry and topology, from: "A symposium in honor of S Lefschetz", Princeton Univ. Press (1957) 280-306 MR0092150 
[15] H Murakami, Y Nakanishi, On a certain move generating link-homology, Math. Ann. 284 (1989) 75-89 MR995383

[16] Y Nakanishi, Y Ohyama, Delta link homotopy for two component links. III, J. Math. Soc. Japan 55 (2003) 641-654 MR1978214

[17] Y Nakanishi, T Shibuya, Link homotopy and quasi self delta-equivalence for links, J. Knot Theory Ramifications 9 (2000) 683-691 MR1762762

[18] Y Nakanishi, T Shibuya, A Yasuhara, Self delta-equivalence of cobordant links, Proc. Amer. Math. Soc. 134 (2006) 2465-2472 MR2213721

[19] T Shibuya, Self $\Delta$-equivalence of ribbon links, Osaka J. Math. 33 (1996) 751-760 MR1424684

[20] T Shibuya, Delta-cobordism and weak self delta-equivalence for links, Mem. Osaka Inst. Tech. Ser. A 52 (2007) 5-9 MR2363559

[21] T Shibuya, Weak self delta-equivalence for links, Kobe J. Math. 24 (2007) 41-52 MR2348019

[22] J Stallings, Homology and central series of groups, J. Algebra 2 (1965) 170-181 MR0175956

[23] A Yasuhara, Self delta-equivalence for links whose Milnor's isotopy invariants vanish, to appear in Trans. Amer. Math. Soc.

Tokyo Gakugei University

Department of Mathematics, Koganeishi, Tokyo 184-8501, Japan

yasuhara@u-gakugei.ac.jp

Received: 6 July 2008 Revised: 26 November 2008 